\title{
NCI CTEP SDC Mesothelioma Sub-Category Terminology
}

National Cancer Institute

\section{Source}

National Cancer Institute. NCI CTEP SDC Mesothelioma Sub-Category Terminology. NCI

Thesaurus. Code C103089.

Mesothelioma is an NCI Cancer Therapeutic Evaluation Program (CT EP) Simplified Disease Classification (SDC) category used to organize cancer-related disease coding that harmonizes with and supports reporting based on the global standard Medical Dictionary for Drug Regulatory Reporting (MedDRA) terminology. 